\title{
Correction to: Effect of copper nanoparticles and organometallic compounds (dibutyltin) on tilapia fish
}

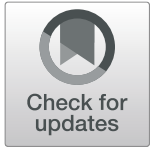

Saif Al Ghais, Vibha Bhardwaj, Pramod Kumbhar and Omar Al Shehhi

\section{Correction to: J Basic Appl Zool https://doi.org/10.1186/s41936-019-0101-7}

Following publication of the original article (Ghais et al., 2019), it was noticed that Figs. 2 and 3 were incomplete.

The correct versions are given below.

Published online: 08 July 2019

\section{Reference}

Ghais, et al. (2019). Effect of copper nanoparticles and organometallic

compounds (dibutyltin) on tilapia fish. The Journal of Basic and Applied Zoology, 80, 32. https://doi.org/10.1186/s41936-019-0101-7.

\footnotetext{
*Correspondence: vibha.bhardwaj1612@yahoo.com

The original article can be found online at https://doi.org/10.1186/s41936019-0101-7

Environment Sustainability Department, Environment Protection and

Development Authority (EPDA), Government of Ras Al Khaimah 11377, UAE
} 


\section{${ }^{-} \mathrm{OOC}-\mathrm{CH}\left(\mathrm{NH}_{3}\right)-\mathrm{CH}_{2}-\mathrm{CH}_{2}-\mathrm{CO}-\mathrm{NH}-\mathrm{CH}\left(\mathrm{CH}_{2}-\mathrm{SH}\right)-\mathrm{CO}-\mathrm{NH}-\mathrm{CH}_{2} \mathrm{COO}^{-}+\mathrm{RSSR} \rightarrow$ (OXIDATION)}

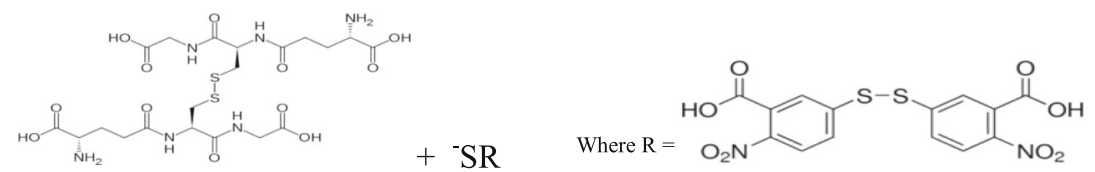

Fig. 2 GSH oxidation with DTNB produce Glutathione disulfide

$$
\begin{array}{ll}
\mathrm{H}_{2} \mathrm{O}+\left(\mathrm{CH}_{3}\right)_{3}-\mathrm{N}^{+}-\mathrm{CH}_{2}-\mathrm{CH}_{2} \mathrm{~S}-\mathrm{COCH}_{3} \rightarrow\left(\mathrm{CH}_{3}\right)_{3}-\mathrm{N}_{-}-\mathrm{CH}_{2}-\mathrm{CH}_{2} \mathrm{~S}^{-}+\mathrm{CHCOO}^{-}+2 \mathrm{H}^{+} \\
\left(\mathrm{CH}_{3}\right)_{3}-\mathrm{N}_{-}-\mathrm{CH}_{2}-\mathrm{CH}_{2} \mathrm{~S}^{-}+\mathrm{RSSR} \rightarrow\left(\mathrm{CH}_{3}\right)_{3}-\mathrm{N}-\mathrm{CH}_{2}-\mathrm{CH}_{2} \mathrm{SSR}+\mathrm{RS}^{-}
\end{array}
$$

Fig. 3 Hydrolysis of thiocholine with DTNB to produce 5-thio-2-nitro benzene ion 